\title{
Emphysematous Splenic Infection in the Setting of Disseminated Klebsiella pneumoniae
}

\author{
Reema Mallick, Carrie Ronstrom, ${ }^{2}$ and Michele Loor ${ }^{1}$
}

\begin{abstract}
Background: Splenic infections present generally in the setting of previous splenic trauma or in patients with relative immune compromise. Primary infections or hematologic spread from remote sites are typical etiologies. Case Presentation: We encountered a 71-year-old diabetic male with history of pancreaticoduodenectomy who presented with Klebsiella bacteremia and a necrotizing infection of the spleen with small, diffuse abscesses throughout the lungs and liver. Imaging was significant for portal venous gas and pneumoperitoneum secondary to the patient's infection. The patient underwent splenectomy and was treated with long-term antibiotic therapy. Diagnostic work-up did not yield a definitive causative etiology other than potential primary infection from one of the affected sites, though colonoscopic evaluation is pending.

Conclusion: Splenic abscesses may be treated with limited drainage or may require splenectomy if multi-focal infection exists.
\end{abstract}

$\mathbf{E}$ MPHYSEMATOUS INFECTION of the spleen is an uncommon clinical entity. Predisposing factors to splenic infection include primary pyogenic infections, trauma, hemoglobinopathies, and contiguous hematologic spread. Here we describe the presentation of a 71-year-old male with manifestations of sepsis secondary to Klebsiella bacteremia and disseminated infection within the liver, lungs, and spleen.

\section{Case Presentation}

A 71-year-old male was hospitalized for fevers, chills, leftsided abdominal pain, vomiting, and diarrhea spanning the previous five days. The patient had a medical history significant for poorly controlled diabetes mellitus type II, stage III chronic kidney disease, and locally advanced pancreatic adenocarcinoma treated successfully with neoadjuvant gemcitabine, radiation, and subsequent pancreaticoduodenectomy (Kausch-Whipple procedure) six years prior. He had done well from treatment for his malignanct disease with negative surgical margins and absence of regional lymph node spread. Recent computed tomography (CT) imaging suggested no evidence of metastatic disease, and the patient was noted to have low CA 19-9 and CEA levels.
On admission, physical examination was significant for altered mental status, diaphoresis, mild dyspnea, and tenderness to palpation in the upper abdominal fields with guarding, more pronounced subcostally on the left side. There were no new cardiac murmurs noted. Laboratory investigations demonstrated a robust inflammatory process with a procalcitonin of 32.88 , lactic acidosis of 3.9 , and leukocytosis of $27.6 \times 10^{3}$. Klebsiella pneumoniae was isolated from initial blood cultures. Liver function tests demonstrated mild elevation of total bilirubin to 1.7 (direct 0.7 ), and mildly elevated transaminases (AST 139; ALT 77). Alkaline phosphatase was increased to 196 , and a urine culture was negative for infection.

A CT scan of the chest, abdomen, and pelvis as well as ultrasound of the abdomen were pursued for further work-up and were notable for multi-focal necrotic cavitary opacities throughout both lungs with concomitant bilateral pulmonary effusions. Imaging of the abdomen demonstrated two hepatic hypodensities with foci of gas, consistent with small abscesses in segments II and VII (approximately 2.7 and $2.2 \mathrm{~cm}$ each in greatest diameter). Most remarkable, however, were the significant emphysematous changes of the spleen with surrounding pneumoperitoneum and portal venous gas, suggestive of

\footnotetext{
${ }^{1}$ Department of Surgery, ${ }^{2}$ School of Medicine, University of Minnesota, Minneapolis, Minnesota.
}

(C) Reema Mallick et al. 2016; Published by Mary Ann Liebert, Inc. This Open Access article is distributed under the terms of the Creative Commons License (http://creativecommons.org/licenses/by/4.0), which permits unrestricted use, distribution, and reproduction in any medium, provided the original work is properly credited. 


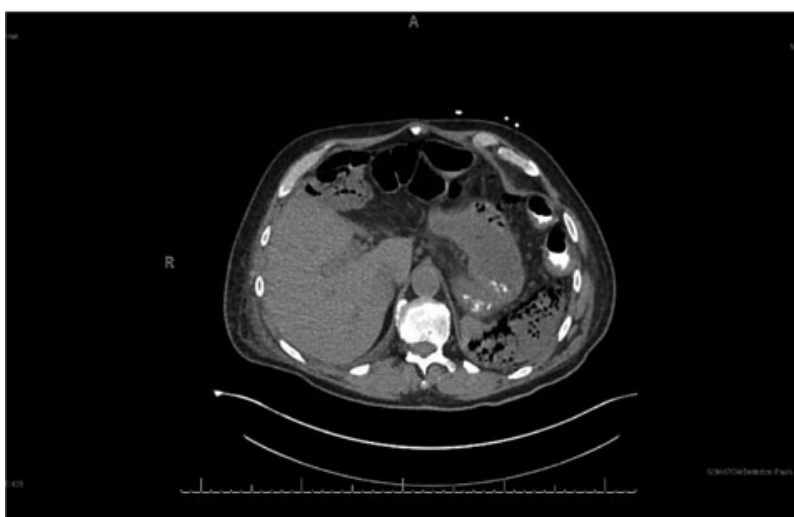

FIG. 1. CT scan of the upper abdomen demonstrating emphysematous changes of the spleen.

necrotizing infection (Fig. 1). The small and large bowel, as well as the hepaticojejunostomy, were otherwise unremarkable.

The patient was initiated on a course of ertapenem pending sensitivities of the $K$. pneumoniae and a continuous insulin infusion for his severe hyperglycemia. His volume status was corrected aggressively. Both transthoracic and transesophageal echocardiograms were pursued to investigate the possibility of a valvular source, but they were ultimately negative. Doppler ultrasound (US) of the abdomen did not demonstrate any source of septic thrombi. Computed tomography imaging of the head was negative for any evidence of septic emboli to account for his lethargy and confusion.

Upon stabilization, the patient was to undergo abdominal exploration with splenectomy. Attempts at embolization of the spleen pre-operatively for ease of vascular control were made by the interventional radiology team, though ultimately this was not feasible secondary to stenosis of the celiac trunk, impeding cannulation.

At laparotomy, the spleen was found to have undergone what appeared to be liquefactive necrosis with a flaccid appearance (Fig. 2). Upon inadvertent capsular entry, there was leakage of a malodorous hematoma. The vessels at the hilum were, at this point, fibrotic. There did not appear to be any evidence for fistulous connection to the spleen from the descending colon or the tail of the pancreas. The hepatic

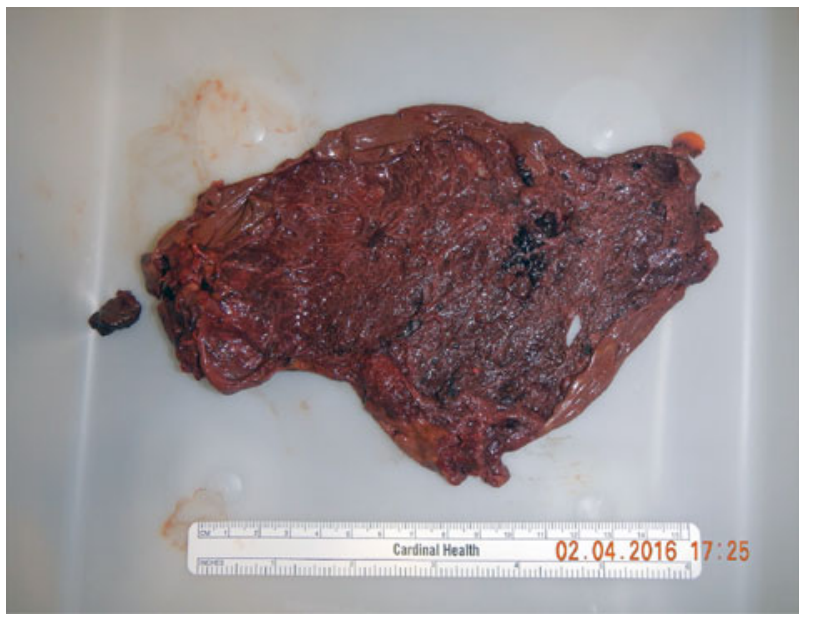

FIG. 2. Spleen with diffuse necrosis and breakdown of structural integrity. abscesses were not grossly visible externally, and exploration of the abdomen did not demonstrate any evidence for intraperitoneal recurrence of the patient's malignant disease. The patient tolerated the procedure well, however, and postoperatively his laboratory markers returned to normal, his mental status improved, and he underwent post-splenectomy vaccinations prior to discharge.

The patient's blood cultures had cleared by the third day of antibiotic therapy, and the patient was discharged on a $6 \mathrm{wk}$ course of IV ceftriaxone with plans for outpatient colonoscopy to eliminate any potential large bowel sources of Klebsiella.

\section{Discussion}

Splenic abscesses and, further, emphysematous infections of the spleen are uncommon clinical findings. This case is particularly unusual given the obscure etiology of the disseminated $K$. pneumoniae. Broadly, splenic infections have been attributed to primary infections, history of recent traumatic injury, hematologic or metastatic spread of infection, and hemoglobinopathies such as sickle cell anemia. Splenic infection is detected more predominantly in patients with damaged splenic parenchyma in association with concomitant malignant disease, immunodeficiency, or diabetes mellitus [1].

Pyogenic infection remains the most common cause of splenic abscesses; however, typical organisms isolated include predominantly gram-negative bacilli such as Escherichia coli, K. pneumoniae, and Proteus mirabilis as well as facultative and aerobic gram-positive bacteria such as Staphlococcus aureus and group D Streptococcus. Up to 55\% of infections in some series have been described as polymicrobial [2]. Endocarditis remains one of the prominent sources of infection (10\%-20\%) and is affiliated more closely with Streptococcus, Staphylococcus, and Klebsiella. Other intra-abdominal conditions have also been associated as primary sources of these infections, including appendicitis, diverticulitis, bowel infarction, or infections of the genitourinary tract, especially with E. coli [2-4].

However, direct invasion of the spleen by means of fistulization of penetrating gastric ulcers, colonic adenocarcinoma at the splenic flexure, or distal pancreatic malignant disease has also been implicated in certain rare instances [56] and can produce the gas-forming necrosis of the spleen demonstrated in the case of this patient.

Concurrent liver abscesses with metastatic spread to the spleen have been detected in other series of patients studied with splenic infection. In one case series, Klebsiella was the major pathogen involved in this finding (100\% of all patients with hepatosplenic involvement). In that cohort, it was presumed that this infection either spread from the liver to the spleen in metachronous fashion versus simultaneous coinfection [7]. Indeed, primary hepatic abscesses secondary to $K$. pneumoniae are being reported with increasing frequency, and older diabetic men such as our patient, tend to be at increased risk. In addition to this risk is increased predisposition to metastatic infection to the eye, central nervous system (CNS), and remote sites secondary to bacteremia [8].

Particular factors that amplify the virulence of these Klebsiella strains include encapsulation of $\mathrm{K} 1$ and $\mathrm{K} 2$ serotypes, magA positivity, a capsule-associated virulence gene, and rmpA, a plasmid-mediated regulator of the extracapsular polysaccharide [8]. 
No clear etiology of this patient's disseminated pathology became evident in the course of our investigation. Primary hepatic abscess in this setting as an initial inciting event is a possibility given the patient's predisposing risk factors, though the presence of gas-producing infection disseminating hematologically from a colonic (or other) source not investigated is also a possibility.

Diagnosis of splenic abscesses is most facile with CT imaging, which is sensitive for this finding (96\%) though there is suggestion that US may be, in experienced settings, equally as diagnostic [3]. Treatment may include CT-guided percutaneous aspiration for unilocular collections or splenectomy for more diffuse infections, as in the case with this patient. For some patients that are not candidates for such therapies, treatment with long-standing antibiotics alone has also been described [5].

\section{Author Disclosure Statement}

The authors have no disclosures to make.

\section{References}

1. Chun CH, Raff MJ, Contreras L, et al. Splenic abscess. Medicine 1980;59:50-65.

2. Brook I, Frazier EH. Microbiology of liver and spleen abscesses. J Med Microbiol 1998;47:1075-1080.

3. Chang KC, Chuah SK, Changchien CS, et al. Clinical characteristics and prognostic factors of splenic abscess: A review of 67 cases in a single medical center of Taiwan. World J Gastroenterol 2006;12:460-464.

4. Chen WH, Yen JC, Kao YF. Disseminated Klebsiella pneumoniae infection after hemorrhoidectomy. Int J Colorectal Dis 2007;22:993-994.

5. Landen S, Closset J. Gas-producing infection of the spleen in a super-super-obese patient. Obes Surg 2007;17:1416-1418.
6. Kawamoto K, Teramoto T, Watanabe M, et al. Splenic abscess associated with colon cancer: A case report. Jpn J Clin Oncol 1993;23:384-388.

7. Lee WS, Choi ST, Kim KK. Splenic abscess: A single institution study and review of the literature. Yonsei Med J 2011;52:288-292.

8. Lee SS, Chen YS, Tsai HC, et al. Predictors of septic metastatic infection and mortality among patients with Klebsiella pneumoniae liver abscess. Clin Infect Dis 2008;47: 642-650.

Address correspondence to:

Dr. Michele Loor

Department of Surgery

University of Minnesota

420 Delaware Street SE, MMC 195

Minneapolis, MN 55455

E-mail: mmloor@umn.edu

Abbreviations Used
$\mathrm{CNS}=$ central nervous system
$\mathrm{CT}=$ computed tomography
$\mathrm{US}=$ ultrasound

Cite this article as: Mallick R, Ronstrom C, Loor M (2016) Emphysematous splenic infection in the setting of disseminated Klebsiella pneumoniae, Surgical Infections Case Reports 1:1, 29-31, DOI: 10.1089/crsi.2016.0004. 